\title{
Early, Subacute Coronary Stent Thrombosis: A Complication of COVID-19 Infection
}

\author{
Sadeq Tabatabai Nooshin Bazargani Kamaleldin Al-Tahmody \\ Jasem Mohammed Alhashmi \\ Cardiology Department, Dubai Hospital, Dubai Health Authority, Dubai, UAE
}

\section{Keywords}

Myocardial infarction - Coronary stent thrombosis .

COVID-19 infection

\begin{abstract}
Soon after it was discovered in Wuhan, China, in December 2019, coronavirus disease 2019 (COVID-19) blow-out very fast and became a pandemic. The usual presentation is respiratory tract infection, but cardiovascular system involvement is sometimes fatal and also a serious personal and health care burden. We report a case of a 57-year-old man who was admitted with anterior wall acute myocardial infarction secondary to early coronary stent thrombosis and associated with COVID-19 infection. He was managed with primary coronary angioplasty and discharged home. Procoagulant and hypercoagulability status associated with severe acute respiratory syndrome coronavirus 2 infection is the most likely culprit. Choosing aggressive antithrombotic agents after coronary angioplasty to prevent stent thrombosis during the COVID-19 pandemic may be the answer but could be challenging.

(C) 2021 The Author(s). Published by S. Karger AG, Basel
\end{abstract}

(C) 2021 The Author(s)

Published by S. Karger AG, Basel

This is an Open Access article licensed under the Creative Common Attribution-NonCommercial-4.0 International License (CC BY-NC) (http://www.karger.com/Services/OpenAccessLicense), applicable to the online version of the article only. Usage and distribution for commercial purposes requires written permission.

\section{Introduction}

Stent thrombosis is an uncommon but most serious complication of coronary artery stenting, usually presented as sudden death or acute myocardial infarction (AMI) if missed or misdiagnosed, which requires immediate revascularization in order to prevent long-term complications [1]. The mechanisms of underlying stent thrombosis are multifactorial, including patient-related factors, procedural factors, and postprocedural factors [2]. In patients with coronavirus disease 2019 (COVID-19), this is even more important than in patients without accompanying diseases.

The usual clinical manifestation of COVID-19 is respiratory symptoms including fever, cough, and shortness of breath. However, since the outbreak in early 2020, different types of cardiovascular involvement and complications of COVID-19 infection have been reported including AMI. Acute ST-elevation myocardial infarction (STEMI) secondary to different types of coronary stent thrombosis ranging from early to very late has been reported in infected patients during the COVID-19 pandemic $[3,4]$.

We present an adult male patient who was admitted to our center with anterior wall AMI secondary to early coronary stent thrombosis associated with COVID-19 infection. He is the first case with such complication admitted to our center during the current pandemic.

Correspondence to:

Sadeq Tabatabai, drsadeq@yahoo.com 
Fig. 1. a Initial ECG showing anterior wall STEMI. b ECG after coronary angioplasty before hospital discharge. c Coronary angiogram showing a total block of LAD (arrow). d LAD flow restored after primary PCI (arrow).
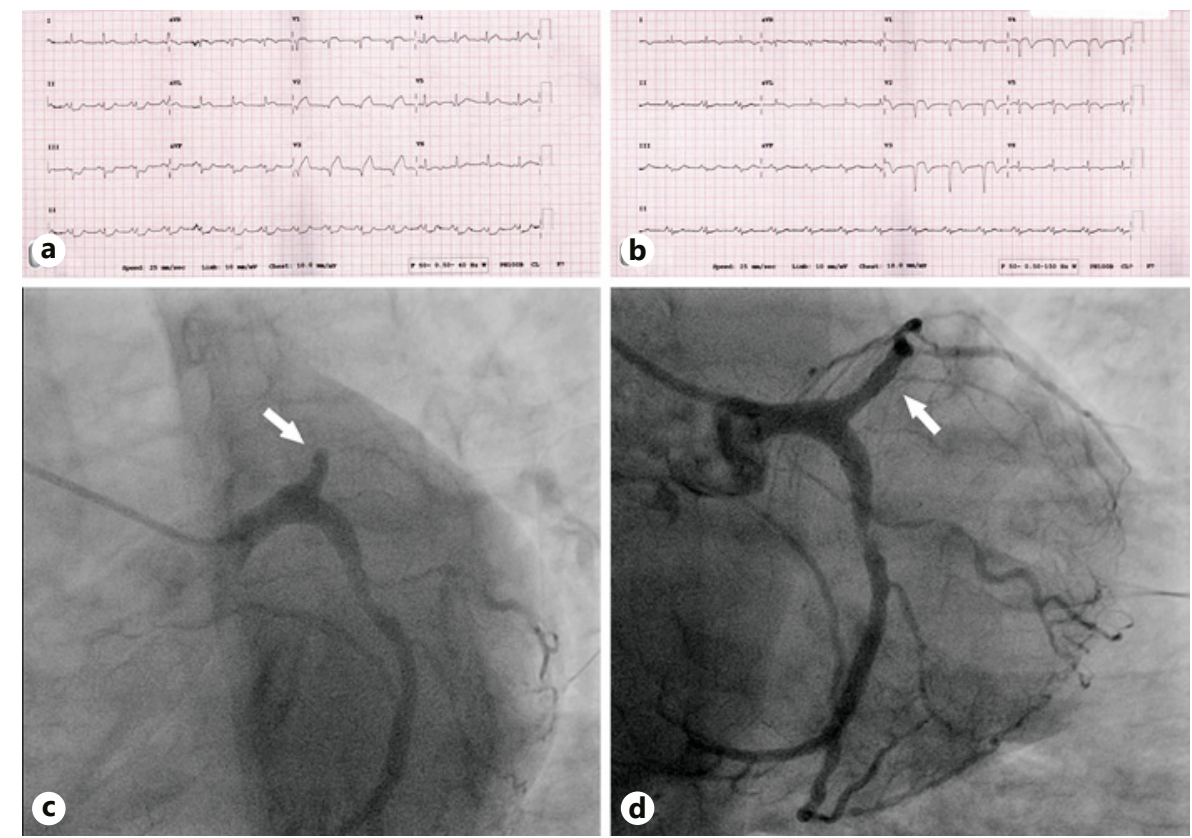

\section{Case Report}

The patient was a 57-year-old male, who presented to the emergency department (ED) at the Dubai hospital for the first time with a history of severe central chest pain radiating to both arms suggestive of acute myocardial ischemia. He had a past medical history including hyperlipidemia, essential hypertension, diabetes mellitus, and ischemic stroke complicated hemorrhagic transformation in the bilateral cerebellar hemisphere and ischemic changes in bilateral fronto-parieto-occipital white matter and chronic thrombosis of the left internal carotid artery. He was kept on dual antiplatelet therapy by his treating physician.

His initial electrocardiogram (ECG) showed evidence of acute anterior wall STEMI, and due to the presence of relative contraindication for medical reperfusion therapy, he was taken for primary percutaneous coronary intervention immediately. Successful coronary angioplasty to the left anterior descending (LAD) artery was performed, and one $3.0 \times 18 \mathrm{~mm}$ Xience Sierra everolimus drug-eluting stent was deployed as shown in Figure 1.

At this stage, severe acute respiratory syndrome coronavirus 2 (SARS-CoV-2) reverse transcriptase polymerase chain reaction assay of the nasopharyngeal swab was negative. His predischarge echocardiogram showed overall left ventricular systolic function is moderately severe impaired with a left ventricular ejection fraction between 30 and 35\%. There was severe hypokinesis of the anterior, antero-septal, and apical walls. The diastolic filling pattern was normal for his age. The patient was discharged in good general condition with dual antiplatelet therapy (aspirin $75 \mathrm{mg}$ daily and clopidogrel $75 \mathrm{mg}$ daily), bisoprolol, ramipril, and a high dose of atorvastatin. Oral hypoglycemic agents were prescribed as well. Around 26 days later, he presented to ED again with severe retrosternal chest pain associated with sweating suggestive of myocardial ischemia. He denied shortness of breath and any other respiratory or gastrointestinal symptoms. The patient indicated that he maintained compliance with the prescribed dual antiplatelet therapy and other postmyocardial infarction medication.

His vital signs on admission showed blood pressure $123 / 93 \mathrm{~mm}$ $\mathrm{Hg}$, pulse $110 \mathrm{bpm}$, temperature $36.9^{\circ} \mathrm{C}\left(98.4^{\circ} \mathrm{F}\right)$, respiratory rate $18 / \mathrm{min}$, height $164 \mathrm{~cm}\left(64.57^{\prime \prime}\right)$, weight $82 \mathrm{~kg}$, and $\mathrm{SpO}_{2} 97 \%$. Initial ECG showed ST-segment elevation in anterior leads suspicious of stent thrombosis. An urgent coronary angiogram confirmed the presence of LAD stent thrombosis. Aspiration catheter was used and thrombectomy performed, and then LAD artery flow was restored with balloon angioplasty using a $3.5 \times 15 \mathrm{~mm}$ noncompliant balloon as shown in Figure 2.

Tirofiban was prescribed after coronary angioplasty, and clopidogrel was replaced with ticagrelor. The patient was discharged alive after completing the isolation period.

At this stage, the SARS-CoV-2 polymerase chain reaction assay of the nasopharyngeal swab was positive. Peak cardiac troponin $\mathrm{T}$ level was 1,483 ng/L, ferritin level $548 \mathrm{ng} / \mathrm{mL}$, D-dimer test $3.42 \mu \mathrm{g} /$ $\mathrm{mL}, \mathrm{C}$-reactive protein $45.8 \mathrm{mg} / \mathrm{L}$, and N-terminal pro B-type natriuretic peptide $4,267 \mathrm{pg} / \mathrm{mL}$. Chest X-ray showed bilateral hilar and para hilar congested vascular markings with no lung consolidations.

\section{Discussion}

Previous research and clinical studies have demonstrated that patients with infectious diseases, both bacterial and nonbacterial like influenza virus infection, have a more intense risk of developing coronary artery thrombosis and AMI by tachycardia, hypoxia, increased wall stress, and activation of systemic coagulation resulting in disseminated intravascular coagulation and thrombocy- 
Fig. 2. a Initial ECG showing anterior wall STEMI due to stent thrombosis. b ECG after primary coronary angioplasty for stent thrombosis. c Coronary angiogram showing LAD stent thrombosis (arrow). d LAD flow restored after thrombectomy and balloon coronary angioplasty for stent thrombosis (arrow).
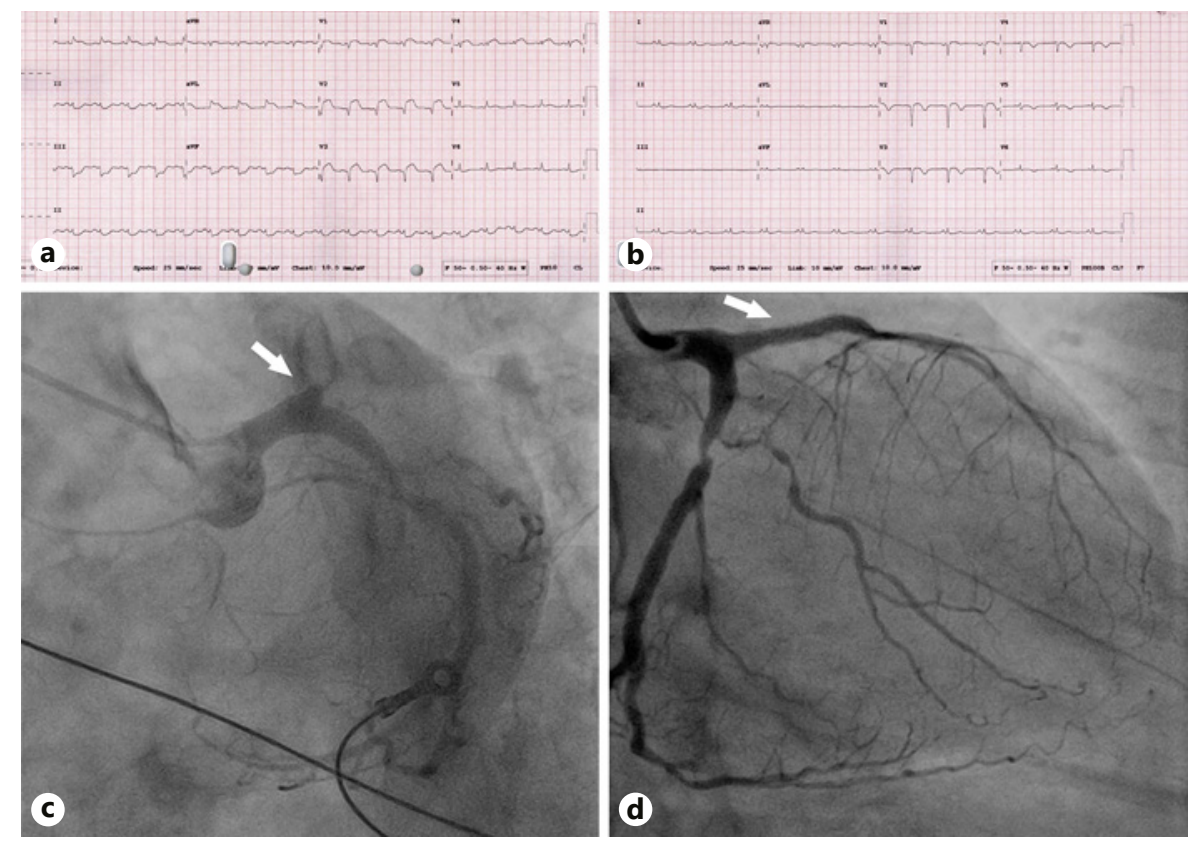

topenia which can lead to multiorgan failure. Although direct interactions between the infectious agent and the coagulation system occur, the release of inflammatory cytokines and endothelial dysfunction, a common feature of viral infection, is believed to be an important key mediator in this process [5].

A recent publication by Canzano et al. [6] provides evidence that the cytokine storm present in COVID-19 patients induces, together with the imbalance of endothelial functions, consistent cell-based tissue factor-mediated activation of coagulation, procoagulant microvesicles release (by activated monocytes and granulocytes) and massive platelet activation causing thrombus formation. These factors predispose the patients with COVID-19 infection to thrombin generation and thrombosis risk, both in the venous and arterial vascular beds, despite prophylactic anticoagulation [6]. Although these patients are at risk of acute coronary events from de novo coronary thrombosis, different studies and registries demonstrated a significant increase in the incidence of acute coronary stent thrombosis in patients with STEMI and SARSCoV-2 infection up to 10 times more likely $[3,7]$.

Hamadeh et al. [8] in a retrospective multicenter, medical chart review of consecutive patients hospitalized with confirmed COVID-19 infection and STEMI included patients from the Middle East (Karbala, Iraq) and reported a $21 \%$ incidence of stent thrombosis among patients who underwent primary percutaneous coronary intervention which is much higher than previously reported rates of early stent thrombosis of $1 \%$ [8]. Hypercoagulability and massive arterial thrombosis including heavy coronary artery thrombus burden have been observed during a coronary angiogram in patients with COVID-19 presented with AMI [9].

In the presence of hypercoagulability status, the prescription of antiplatelet therapy after coronary angioplasty for stent thrombosis prevention is challenging. In combination with aspirin, some reports support the use of ticagrelor as a potent P2Y12 inhibitor [4], while others prefer the use of prasugrel [3]. Triple antithrombotic treatment with low-dose rivaroxaban is worth to be considered [10]. Of course, probably we need more data from randomized trials and large registries to confirm the optimal antithrombotic treatment in such patients.

We assume that the main issue in our patient is the high tendency to develop a highly thrombotic environment and increased thrombogenicity secondary to various factors, enhancing coagulopathy which led to an increase in the risk of acute coronary syndrome and precipitates stent thrombosis despite up-to-date optimal dual antiplatelet medical therapy.

Stent under expansion and clopidogrel resistance are another possible cause for early stent thrombosis in our patient, but unfortunately, intravascular ultrasound or coronary optical coherence tomography was not done, and a test to detect clopidogrel resistance is not available in our center. Nonetheless, given this is a single case report, we are unable to conclude that the early stent throm- 
bosis was caused by COVID-19 illness. This also prohibits our findings from being generalizable to additional COVID-19 patients. These findings, however, imply that extra attention should be devoted to optimizing antithrombotic treatment in COVID-19 patients with prior coronary artery disease.

\section{Conclusion}

This gentleman suffered from AMI secondary to coronary stent thrombosis. We propose that the stent thrombosis in our patient may be precipitated by the procoagulant and hypercoagulability status secondary to acute COVID-19 infection. We need data from large registries to confirm this suspected increased risk of this complication in these patients combining STEMI and COVID-19 infection. This report aims mainly to highlight this particular situation that may become more and more frequent during this pandemic.

\section{Statement of Ethics}

This case report complies with the guidelines for human studies and was conducted ethically in accordance with the World Medical Association Declaration of Helsinki. Ethical approval was not required for this case report in accordance with the Dubai Scientific Research Ethics Committee policies. Written informed consent for publication of the case report and any accompanied images was obtained from the patient.

\section{Conflict of Interest Statement}

The authors declared no potential conflicts of interest with respect to the research, authorship, and/or publication of this article.

\section{Funding Sources}

The authors received no financial support for the research, authorship, and/or publication of this article.

\section{Author Contributions}

All the authors contributed equally in writing the manuscript. All authors read and approved the final manuscript.

\section{Data Availability Statement}

All data generated or analyzed during this study are included in this article. Further inquiries can be directed to the corresponding author.

\section{References}

1 Varenhorst C, Lindholm M, Sarno G, Olivecrona G, Jensen U, Nilsson J, et al. Stent thrombosis rates the first year and beyond with new- and old-generation drug-eluting stents compared to bare metal stents. Clin Res Cardiol. 2018;107(9):816-23.

2 Ullrich H, Münzel T, Gori T. Coronary stent thrombosis: predictors and prevention. Dtsch Arztebl Int. 2020;117(18):320-6.

3 Prieto-Lobato A, Ramos-Martínez R, VallejoCalcerrada N, Corbí-Pascual M, Córdoba-Soriano JG. A case series of stent thrombosis during the COVID-19 pandemic. JACC Case Rep. 2020;2(9):1291-6.

4 Choudhary R, Kaushik A, Sharma JB. COVID-19 pandemic and stent thrombosis in a post percutaneous coronary intervention patient-a case report highlighting the selection of P2Y12 inhibitor. Cardiovasc Diagn Ther. 2020;10(4):898-901.
5 Barnes M, Heywood AE, Mahimbo A, Rahman B, Newall AT, Macintyre CR. Acute myocardial infarction and influenza: a metaanalysis of case-control studies. Heart. 2015; 101(21):1738-47.

6 Canzano P, Brambilla M, Porro B, Cosentino $\mathrm{N}$, Tortorici E, Vicini S, et al. Platelet and endothelial activation as potential mechanisms behind the thrombotic complications of $\mathrm{CO}$ VID-19 patients. JACC Basic Transl Sci. 2021; 6(3):202-18.

7 Choudry FA, Hamshere SM, Rathod KS, Akhtar MM, Archbold A, Guttmann OP, et al. High thrombus burden in patients with COVID-19 presenting with ST-segment elevation myocardial infarction. J Am Coll Cardiol. 2020;76(10):1168-76.
8 Hamadeh A, Aldujeli A, Briedis K, Tecson KM, Sanz-Sánchez J, Al Dujeili M, et al. Characteristics and outcomes in patients presenting with COVID-19 and ST-segment elevation myocardial infarction. Am J Cardiol. 2020;131:1-6.

9 Seif S, Ayuna A, Kumar A, Macdonald J. Massive coronary thrombosis caused primary percutaneous coronary intervention to fail in a COVID-19 patient with ST-elevation myocardial infarction. Catheter Cardiovasc Interv. 2021;97(5):E667-9.

10 Al-Sadawi M, Mohiuddin A, Hossain N, Shaikh S, Feit A, Ramalanjaona B, et al. Management of ST-elevation myocardial infarction in the COVID-19 era: the role of thrombosis and anticoagulation strategy. Am J Med Case Rep. $2020 ; 8(9): 262-7$ 